\title{
Acute ophthalmoparesis and persistent mydriasis: expanding the clinical spectrum of anti-GQ1b positive cranial neuropathy in a 5.5-year-old girl
}

\author{
Ayfer Sakarya Güneş ${ }^{1}$, Hülya Maraş Genç ${ }^{1}$, Emek Uyur Yalçın, Vuslat Yılmaz², \\ Güher Saruhan Direskeneli ${ }^{2}$, Bülent Kara ${ }^{1}$ \\ ${ }^{1}$ Division of Child Neurology, Department of Pediatrics, Kocaeli University Faculty of Medicine, Kocaeli; ${ }^{2}$ Department of \\ Physiology, Istanbul University Faculty of Medicine, Istanbul, Turkey. E-mail: ayfer-sakarya@hotmail.com \\ Received: 29th May 2018, Revised: 18th September 2018, 27th November 2018, \\ Accepted: 15th December 2018
}

SUMMARY: Sakarya Güneş A, Maraş Genç H, Uyur Yalçın E, Yılmaz V, Saruhan Direskeneli G, Kara B. Acute ophthalmoparesis and persistent mydriasis: expanding the clinical spectrum of anti-GQ1b positive cranial neuropathy in a 5.5-year-old girl. Turk J Pediatr 2019; 61: 794-797.

Acute ophthalmoparesis without ataxia (AO) is an atypical form of MillerFisher syndrome (MFS) and is rare in children. Anti-GQ1b antibodies can be detected in patients with AO, as in MFS. A 5.5-year-old girl had total ophthalmoparesis, blurred vision, ptosis, diplopia and mydriasis non-reactive to light or near stimuli with preserved consciousness and deep tendon reflexes. She had no ataxia. Cerebrospinal fluid (CSF) examination and cranial MRI were normal. Serum antiGQ1b antibodies were positive. She was diagnosed with AO and intravenous Immunoglobulin (IVIG) was ordered, $400 \mathrm{mg}$ / $\mathrm{kg} / \mathrm{day}$, for 5 days. Ophthalmoparesis and blurred vision improved in a few weeks. At the end of the first year, mydriasis still persisted, but improved and became responsive to near stimuli. Pupillary involvement may be seen in approximately $50 \%$ of MFS patients, and improvement in a few weeks or months has been reported in adults. Our case shows the expanding clinical spectrum of anti-GQ1b positive cranial neuropathy as early-onset AO and prolonged mydriasis more than one year.

Key words: acute ophthalmoparesis without ataxia, acute ophthalmoparesis, MillerFisher syndrome, anti-GQ1b; mydriasis.

Miller-Fisher syndrome (MFS) is a polyradiculoneuropathy, characterized by the clinical triad of acute-onset ophthalmoparesis, ataxia and areflexia. ${ }^{1}$ It is considered as a variant of Guillain-Barrè syndrome (GBS) because of the similar immuno-pathogenesis. ${ }^{1}$

Most of patients with MFS have anti-GQ1b antibodies. $^{2}$

Acute ophthalmoparesis without ataxia (AO) and acute ataxic neuropathy without ophthalmoparesis are atypical forms of MFS and are rarely observed during childhood. ${ }^{2}$ Here, we present the one year follow-up of a 5.5-year-old girl who had acute bilateral complete external ophthalmoparesis, mydriasis and high serum anti-GQ1b antibodies with the diagnosis of $\mathrm{AO}$.

\section{Case Report}

A 5,5-year-old girl was brought to the emergency department with acute diplopia, bilateral ptosis and bilateral mydriasis that had started four days ago. She had a cough and

This manuscript was published in abstract form of the 18th National Child Neurology Congress, 20-24 April 2016, Antalya, Turkey. 
nasal discharge which started seven days ago. The diplopia and ptosis displayed no diurnal variation, and diplopia disappeared with closure of either eye. There was no history of fever, changes in consciousness, seizures, facial asymmetry, difficulty in chewing or swallowing and gait abnormalities. She was born at term and, prenatal, natal and early postnatal histories were uneventful. She had no similar past events, head trauma or toxic substance exposure. Developmental milestones were normal. There was no similar disease in the family. At first examination, her weight was $20 \mathrm{~kg}$ (50-75th percentile), height $113 \mathrm{~cm}$ (5075 th percentile) and vital signs were stable. Her consciousness was clear and cognition was normal. She had near complete (vertical and horizontal) bilateral ophthalmoparesis with ptosis and blurred vision. The pupils were extremely mydriatic and non-reactive to light or near stimuli. Cholinergic hypersensitivity could not be tested. All other cranial nerves and ocular fundus examinations were intact. The muscle power of proximal and distal muscles of all extremities were normal. Deep tendon reflexes were normal, and plantar responses were flexor. Sensory examination was not optimal. Cerebellar signs were negative. The rest of the physical examination was normal.

Whole blood count, CRP, erythrocyte sedimentation rate, serum electrolytes, renal and hepatic function tests and urine analysis were normal. The cerebrospinal fluid (CSF) analysis revealed normal protein $(15.1 \mathrm{mg} /$ dl) and glucose $(58 \mathrm{mg} / \mathrm{dl})$ levels without pleocytosis. The gadolinium-enhanced magnetic resonance imaging (MRI) of the brain and orbita were normal. Electromyography could not be performed at disease onset. Serum anti-nuclear antibody test was negative and plasma levels of complement proteins (C3, C4) were normal. Thyroid function tests were normal with negative thyroid autoantibodies. On the 5th day of hospital admission, serum antiganglioside antibody panel was ordered and serum anti-GQ1b IgG antibody was found to be positive (titres 1:290) and other antiganglioside antibodies (anti-GM1, -GM2, -GM3, -GD1a, -GD1b and -GT1b) were negative.

The patient was diagnosed with acute ophthalmoparesis without ataxia, a rare form of MFS and intravenous immune globulin (IVIG) was ordered, totally $2 \mathrm{~g} / \mathrm{kg}$ for five days. During the first weeks of treatment, diplopia, ptosis, vertical gaze palsy and blurred vision improved, but dilated pupils persisted. At the 4th month of the follow-up, the patient had normal eye movements in all directions, but her pupils were moderately mydriatic and non-reactive to light or near stimuli. Electromyographic examination showed mildly reduced sensorial nerve conduction velocities at the upper extremities, and the rest of the examination was normal, but the needle examination could not be done. At the 12th month follow-up, the patient had photophobia and her pupils were still mydriatic, but light and near stimuli response became mildly positive. Informed consent was received from the family.

\section{Discussion}

Miller-Fisher syndrome (MFS) was first described in 1956 as an atypical variant of GBS with the clinical triad of acute-onset ophthalmoparesis, ataxia and areflexia. ${ }^{1}$ It is very rare during childhood and its incidence is not known, however, its annual incidence is estimated to be less than one in million. ${ }^{2}$ Similar to GBS, post-infectious immunological mechanisms are responsible. Therefore, difficulties occur in the differential diagnosis of GBS, MFS, polyneuritis cranialis and Bickerstaff's brainstem encephalitis (BBE), which all develop via similar mechanisms and clinical manifestations.

Although immunologic studies detect different antibodies, diagnostic difficulties still exist. Acute ophthalmoparesis without ataxia (AO) is a rare variant of MFS. AO is more frequent in adulthood and mixt type (horizontal and vertical) ophthalmoparesis is the most frequent clinical finding and binocular involvement is common. Progressive, symmetric ophthalmoparesis up to 4 weeks and absence of ataxia, areflexia and limb weakness are the mandatory criteria for the diagnosis $\mathrm{AO}^{3}$.

Antibodies to epitopes located in the axolemma and the myelin sheath (GT1b, GD1b, GM1, GT1a, GD1a, etc.) are produced in MFS, while anti-GQ1b is the most commonly detected 
antibody. During the acute period, anti-GQ1b antibodies are detected at a rate of $95 \%$ and known as a biomarker. ${ }^{2,4}$ In relation to MFS, positive anti-GQ1b antibodies also highly confirms the diagnosis of incomplete forms of MFS, such as AO and acute ataxic neuropathy without ophthalmoparesis (AAN). AO was diagnosed with a frequency of $6 \%$ in antiGQ1b antibody-positive patients. ${ }^{5}$ However, anti-GQ1b antibodies can be detected in GBS, Bickerstaff's brainstem encephalitis and pharyngeal-cervical-brachial variants of GBS. In association with the immunologic properties of anti-GQ1b antibody, highly variable clinical manifestations can be observed due to the involvement of different cranial nerves, particularly ophthalmologic findings such as optic neuritis, areflexic mydriasis, convergence defect, divergence paralysis, internal ophthalmoparesis, and chronic ophthalmoparesis. It may also present with isolated abducens paralysis. ${ }^{2}$ It is still unclear why anti-GQ1b antibody is associated with such a wide clinical spectrum. One of the possible determinants seems to be the reactivity of the autoantibodies to the ganglioside complex. ${ }^{5}$ Internal ophtalmoparesis (mydriasis) could lead to severe blurred vision. ${ }^{6}$ Optic nerve involvement may also be seen in the clinical spectrum of anti-GQ1b positive patients, but the cause of visual problems in our case was probably due to mydriasis. Despite lack of visual evoked potential (VEP) test, our hypothesis is supported by normal eye fundus examination and gadolinium-enhanced magnetic resonance imaging of the orbita.

The main clinical findings of our patient were post-infectious, symmetric, combined external and internal ophtalmoparesis, preserved deep tendon reflexes and absence of ataxia. Botulismus was excluded because there was no history of recent ingestion of honey, preserved or canned food, and no clinical findings of descending paralysis. Normal brain MRI was not compatible with brainstem lesions (tumor, demyelinating lesion, vascular, inflammatory, etc.) which usually result in asymmetric ophtalmoparesis. Myasthenia gravis should also be among the differential diagnosis for external ophtalmoparesis, but the existence of internal ophtalmoparesis and lack of fluctuating clinical symptoms and specific electrophysiologic findings, eliminate myasthenia gravis. After obtaining positive anti-GQ1b result, the differential diagnosis was limited for variants of GBS. MFS (no areflexia and ataxia), polyneuritis cranialis (no bulbar symptoms), and Bickerstaff brainstem encephalitis (no loss of conscious and hyperreflexia) were all excluded by clinical findings. Clinical findings of our patient were mostly compatible with $\mathrm{AO}$, and fulfilled all the diagnostic criteria defined by Odaka et al. ${ }^{3}$

Studies have shown that anti-GQ1b antibody was significantly correlated only with ophthalmoparesis. ${ }^{5} \quad$ Several mechanisms have been proposed to explain the role of anti-GQ1b antibody in development of AO. GQ1b ganglioside is abundantly present in the paranodal and neuromuscular junction areas of trochlear and abducens nerves and mostly in the oculomotor sites. This finding supports development of ophthalmoparesis as a major symptom in MFS, and its variants. ${ }^{7}$ Additionally, immune-histochemical studies in MFS patients revealed that the anti-GQ1b antibody was detected at high levels in the III, IV and VI cranial nerves. To support the involvement of these nerves, marked gadolinium uptake was shown on gadoliniumenhanced MRI images. ${ }^{8}$

Pupillary involvement can occur in approximately $50 \%$ (14\%-84\%) of patients with MFS or its variants, and mydriasis is the most frequent finding. ${ }^{9}$ Both fixed dilated pupils reactive to neither light nor near stimuli (accommodation) due to involvement of preganglionic parasympathetic pathway and tonic pupils due to damage of the ciliary ganglion or short ciliary nerves are reported in MFS and its variants. ${ }^{9}$ Tonic pupil (Adie pupil) does not react to light, but shows some near response (light-near dissociation), and has high pilocarpine (\%0.1) hypersensitivity. It usually results in unilateral involvement, and recovery is incomplete. Nitta et al. ${ }^{10}$ reported 4 patients with mydriasis due to MFS, two of them had tonic pupils and others fixed dilated pupils. We could not test for the cholinergic hypersensitivity, but the absence of light-near dissociation, prolonged nonreactive pupils to near stimuli and bilateral involvement supported the pathogenesis of mydriasis as fixed dilated pupils. 
External and internal ophtalmoparesis are selflimited and have good prognosis. ${ }^{8}$ External ophtalmoparesis usually recover in a shorter time than internal ophtalmoparesis. There is no evidence-based data on the treatment of acute ophtalmoparesis in children. Because of the favorable natural history of this disorder, it is difficult to determine treatment efficacy. Despite spontaneous recovery, immunological therapies might be a considerable alternative, because of severe visual problems which may prolong from weeks to months. Sato et al. ${ }^{6}$ reviewed 7 adult patients with isolated internal ophtalmoparesis (mydriasis), and reported that steroids were not beneficial. Due to the similar associations in etiology and pathogenesis with typical GBS; IVIG and plasmapheresis therapies can also be considered for acute ophthalmoparesis. But, it should be kept in mind that, complete spontaneous recovery is a rule, and these therapies might only hasten recovery. In our patient, we preferred to use IVIG to shorten the duration of visual problems which decreases quality of life. It is controversial to determine the effect of IVIG for external ophtalmoparesis, but it is clear that IVIG was not beneficial for internal ophtalmoparesis in our patient.

Our case shows the expanding clinical spectrum of anti-GQ1b positive cranial neuropathy as the early-onset acute ophthalmoparesis without ataxia (AO) and the prolonged mydriasis more than one year. The natural favorable prognosis of acute external ophtalmoparesis complicates our ability to evaluate the efficacy of IVIG, however, we did not observe any beneficial effect of IVIG for internal ophtalmoparesis in our case.

\section{REFERENCES}

1. Fisher M. An unusual variant of acute idiopathic polyneuritis. N Engl J Med 1956; 255: 57-65.

2. Lo YL. Clinical and immunological spectrum of the Miller Fisher syndrome. Muscle Nerve 2007; 36: 615627

3. Odaka M, Yuki N, Hirata K. Anti-GQ1b IgG antibody syndrome: clinical and immunological range. J Neurol Neurosurg Psychiatry 2001; 70: 50-55.

4. Lee SH, Lim GH, Kim JS, et al. Acute ophthalmoplegia (without ataxia) associated with anti-GQ1b antibody. Neurology 2008; 71: 426-429.

5. Fukami Y, Wong AHY, Funakoshi K, Safri AY, Shahrizaila N, Yuki N. Anti-GQ1b antibody syndrome. Eur J Neurol 2016; 23: 320-326.

6. Sato H, Naito K, Hashimto T. Acute isolated bilateral mydriasis. Case reports and review on the literature. Case Rep Neurol 2014; 6: 74-77.

7. Liu JX, Willison HJ, Pedrosa-Domellöf F. Immunolocalization of GQ1b and related gangliosides in human extraocular neuromuscular junctions and muscle spindles. Invest Ophthalmol Vis Sci 2009; 50: 3226-3232.

8. Nagaoka U, Kato T, Kurita $\mathrm{K}$, et al. Cranial nerve enhancement on three-dimensional MRI in Miller Fisher syndrome. Neurology 1996; 47: 1601-1602.

9. Kaymakamzade B, Selcuk F, Koysuren A, Colpak AI, Mut SE, Kansu T. Pupillary involvement in Miller Fisher syndrome. Neuro-Ophthalmol 2013; 37: 111115.

10. Nitta T, Kase M, Shinmei Y, Yoshida K, Chin S, Ohno S. Mydriasis with light-near dissociation in Fisher's syndrome. Jpn J Ophthalmol 2007; 51: 224-227. 